\title{
SELECTIVE AND REVERSIBLE DISRUPTION OF MITOCHONDRIAL INNER MEMBRANE PROTEIN COMPLEXES BY LIPOPHILIC CATIONS
}

\author{
Anezka Kafkova ${ }^{1}$, Lisa Tilokani ${ }^{2}$, Filip Trčka ${ }^{1}$, Veronika Šrámková ${ }^{3}$, Marie Vancová ${ }^{4,5}$, Tomáš Bílý ${ }^{4,5}$, Jana \\ Nebesařová ${ }^{4,6}$, Julien Prudent ${ }^{2}$, Jan Trnka ${ }^{1 *}$
}

\footnotetext{
${ }^{1}$ Laboratory for Metabolism and Bioenergetics, Department of Biochemistry, Cell and Molecular Biology, Third Faculty of Medicine, Charles University, Czech Republic

${ }^{2}$ Medical Research Council Mitochondrial Biology Unit, University of Cambridge, Cambridge Biomedical Campus, Cambridge CB2 OXY, UK.

${ }^{3}$ Department of Pathophysiology, Third Faculty of Medicine, Charles University, Prague, Czech Republic.

${ }^{4}$ Institute of Parasitology, Biology Centre of the Czech Academy of Sciences, Ceske Budejovice, Czech Republic.

${ }^{5}$ Faculty of Science, University of South Bohemia, Ceske Budejovice, Czech Republic.

${ }^{6}$ Laboratory of Electron Microscopy, Faculty of Science, Charles University, Prague, Czech Republic.
}

*corresponding author: 3. LF UK, Ruska 87, Praha 10, 100 00, Czech Republic, e-mail: jan.trnka@If3.cuni.cz

KEY WORDS: mitochondria, inner mitochondrial membrane, lipophilic cations, membrane potential, mitochondrial dynamics, respiratory chain, mitochondrial drug delivery

\begin{abstract}
Mitochondria represent an attractive drug target in the treatment of many diseases. One of the most commonly used approaches to deliver therapeutics specifically into mitochondria is their conjugation to the triphenylphosphonium (TPP) moiety. While the TPP molecule is often regarded as biologically inert, there is evidence that the moiety itself has a significant impact on the activity of mitochondrial respiratory chain complexes.
\end{abstract}

We studied the impact of a subchronic exposure of C2C12 mouse myoblasts to a set of TPP derivatives. Our results show that the alkyl-TPP cause dose- and hydrophobicity-dependent alterations of mitochondrial morphology and a selective decrease in the amounts of mitochondrial inner membrane (but not outer membrane) proteins including structural subunits of the respiratory chain complexes (such as MT-CO1 of complex IV or NDUFB8 of complex I), as well as components of the mitochondrial calcium uniporter complex (MCUC). The treatment with alkyl-TPP additionally resulted in OPA1cleavage. Both the structural and functional effects of alkyl-TPP were found to be reversible. A similar effect was observed with the mitochondria-targeted antioxidant MitoQ. We further show that this effect on protein levels cannot be explained solely by a decrease in mitochondrial membrane potential.

We conclude that TPP derivatives negatively affect mitochondrial structure and function at least in part through their effect on selective mitochondrial membrane protein levels via a reversible controlled process.

\section{INTRODUCTION}


Mitochondria are organelles involved in a range of functions critical for cell survival ${ }^{1}$. Their functional versatility is accompanied by the remodelling of their network, referred to as mitochondrial dynamics ${ }^{2}$. These dynamic processes encompass continuous cycles of fission and fusion events, which is crucial to answer cellular needs and to adapt to the metabolic state.

Mitochondria are delimited by two membranes, a relatively permeable outer mitochondrial membrane (OMM) and a highly invaginated and selectively permeable inner mitochondrial membrane (IMM). The IMM is highly folded into cristae and contains range of protein complexes, including those regulating cristae shape, but also respiratory chain complexes, ATP synthase or complexes involved in the regulation of calcium homeostasis (mitochondrial calcium uniporter complex, MCUC, or mitochondrial sodium calcium exchanger, $\mathrm{NCLX})^{34}$. Mitochondrial respiratory chain complexes are responsible for electron transport and the generation of a proton gradient across the IMM, which is used by ATP synthase to produce ATP5 .

Mitochondria play an important role in many human diseases and being able to manipulate directly their function through targeted drug delivery may be of a substantial benefit ${ }^{1}$. Delivering drugs specifically into mitochondria is challenging because of the double lipid bilayers and the selective permeability of the $\mathrm{IMM}^{6}$. However, several strategies for targeting small molecule drugs directly to the mitochondria have been successfully developed. One of the most versatile approaches is to conjugate a small bioactive compound to lipophilic cations such as triphenyl phosphonium (TPP) ${ }^{7}$. These were originally used for measuring of the membrane potential but they have evolved as a targeting strategy for drug delivery ${ }^{8}$.

The chemical properties of the positively charged TPP molecule, combined with the large negative membrane potential in the mitochondrial matrix, enables its free movement through the phospholipid bilayer without relying on a specific uptake mechanism. The three hydrophobic phenyl groups on the TPP moiety shield the positive charge on the phosphorus atom from water molecules to prevent solvation, which lowers the energy, preventing the partitioning of the cation into the hydrophobic core of the membrane. At the same time, since the membrane potential across the IMM is up to three time higher compared to the cytoplasm, TPP-conjugated molecules accumulate according to the Nernst equation inside mitochondria ${ }^{9}$. The uptake of the more hydrophobic TPP cations, such as hexyl or decyl-TPP, is more extensive and faster compared to methyltriphenylphosphonium (TPMP), in which the TPP cation is substituted only with a methyl group ${ }^{10}$.

In comparison to unconjugated molecules, multiple studies have shown an increased efficacy of drugs conjugated to TPP 111213141516 , as well as their potential side effects have been investigated ${ }^{171819}$. MitoQ, a known antioxidant conjugated to decyl-TPP, is already undergoing clinical trials and is close to clinical practice ${ }^{20}$. However, the consequences of TPP mitochondria-targeting moiety itself on different basic mitochondrial behaviour are currently not well characterised. Although the TPP moiety is often seen as inert, several studies have described its significant biological effects, particularly in the inhibition of respiratory chain complexes ${ }^{1921}$, enzymes of the Krebs cycle, ${ }^{22}$ or the $\mathrm{NCLX}^{18}$. The underlying mechanisms of these effects as well as other possible effects on mitochondrial morphology and IMM architecture are still largely unknown.

This study investigates the effect of the alkyl substituted TPP moiety on mitochondrial morphology as well as the stability and turnover of different mitochondrial protein complexes, their impact on mitochondrial bioenergetics and reversibility of these processes. We show that TPP treatment for 24 hours at $2 \mu \mathrm{M}$ leads to a significant and selective downregulation of different complexes localized at the IMM including complexes of the respiratory chain (RC) and the MCUC. These reversible effects on IMM complexes were accompanied by a decrease of mitochondrial membrane potential and defects 
in mitochondrial bioenergetics. Finally, confocal and cryo-electron microscopy analysis reveal that TPP treatment leads to a dramatic remodelling of the mitochondrial network characterized by not only mitochondrial fragmentation and swelling, but also by IMM structure alterations.

\section{RESULTS}

1. TPP derivatives affect OXPHOS complex protein levels in a dose-, time- and hydrophobicitydependent manner

Previous studies have shown that different alkylated TPP cations inhibit cellular respiration after only a few minutes of exposure ${ }^{1921}$. These studies were exclusively focused on functional analyses and did not provide any evidence of possible structural defects of the mitochondrial respiratory chain induced by the TPP molecules or the potential effects of longer-term treatment. To address these issues, we exposed $\mathrm{C} 2 \mathrm{C} 12$ cells to three alkyl-TPP derivatives with an increasing alkyl chain length and increasing hydrophobicity, namely methyltriphenylphosphonium (TPMP), propyltriphenylphosphonium (propylTPP or 3-TPP) and hexyltriphenylphoshphonium (hexyl-TPP or 6TPP, for structures see Fig. S1A-C), for 24 hours and analysed the effect on steady state protein levels of components of OXPHOS complexes including MTCO1 for CIV and SDHB for CII (Fig. 1A-C, quantification in Fig. S2A-C).

Incubation with the compounds led to a concentration- and hydrophobicity-dependent decrease in the levels of inner mitochondrial membrane proteins; the subunits of complex IV (MT-CO1) and complex II (SDHB). While hexyl-TPP caused a strong reduction of protein levels at $2 \mu \mathrm{M}, 15$ and $40 \mu \mathrm{M}$ of propyl TPP and TPMP, respectively, had to be used to achieve a similar decrease in the amounts of respiratory chain subunits (Fig. 1D). This is in accordance with the literature Ross et al. ${ }^{10}$ where increasing the hydrophobicity of TPP compounds (i.e. the length of alkyl chain) correlates with an increased ability of TPP derivatives to cross the plasma and mitochondrial membranes which facilitate their accumulation in mitochondria. We used mainly the most active compound (hexyl-TPP) in further studies.
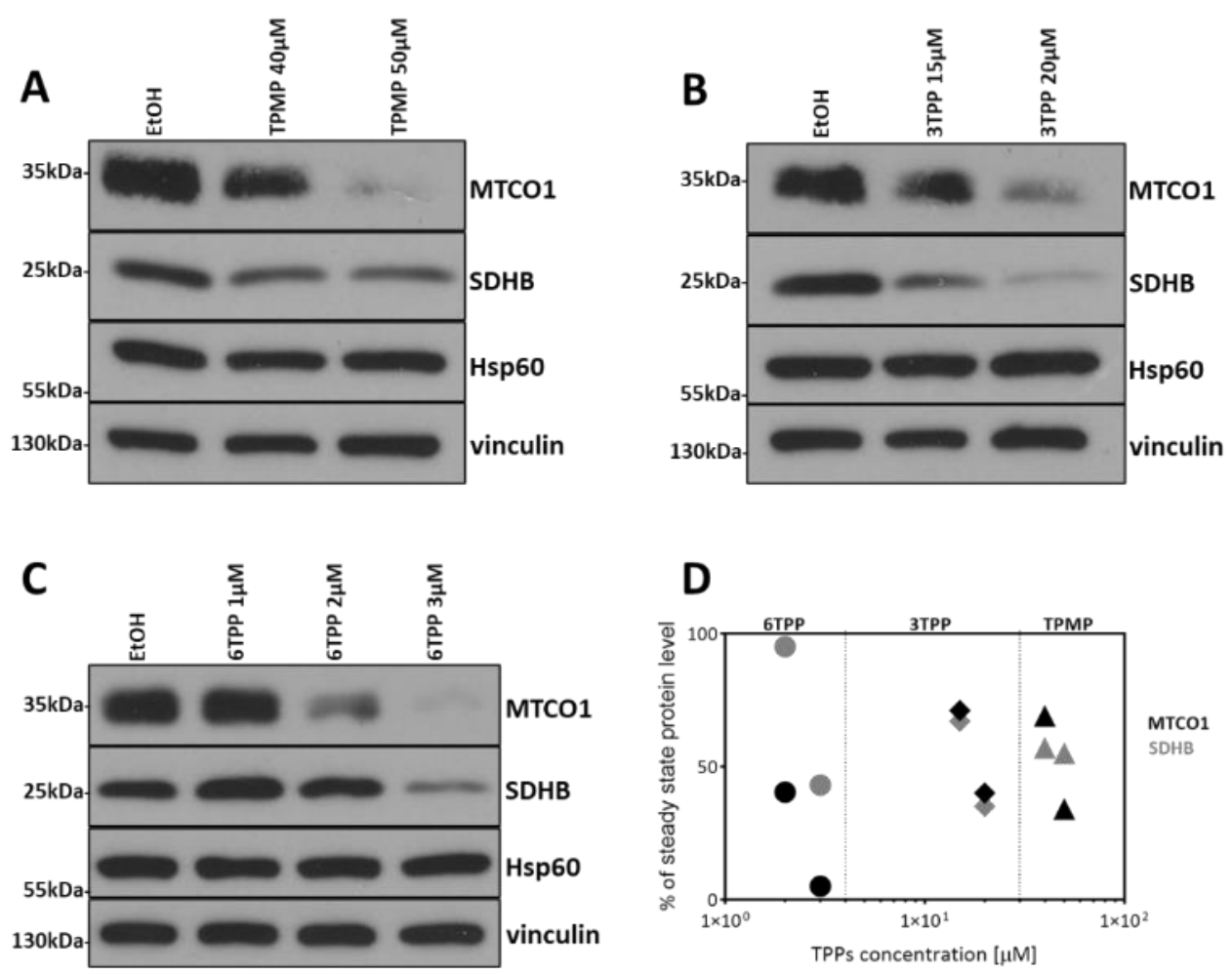
Figure 1. Treatment with TPP derivatives leads to a decrease in selected subunits of OXPHOS complexes. Representative immunoblot for steady-state levels of respiratory chain complex IV (anti-MTCO1) and complex II (anti-SDHB) from C2C12 cells treated for 24 hours with ethanol (EtOH) or different concentration of hexyl-TPP (6-TPP) (A), propyl-TPP (3-TPP) (B), and methyltriphenylphosphonium (TPMP) (C). Vinculin and Hsp60 were used as loading controls. Scans of original immunoblots from A-C can be found in Fig. S6. D. The percentage reduction in steady state protein levels upon exposure to TPPs with different hydrophobicity (compared to vehicle, $x$-axis log scale).

Next, we analysed the effect of hexyl-TPP treatment on the steady-state protein levels of one representative subunit of each respiratory complex, CI (NDUFB8), CII (SDHB), CIII (COREI), CIV (MTCO1) as well as of ATP synthase (ATP5a) at different timepoints (Fig.2A, quantification Fig. S3A). While the levels of ATP5a remained unchanged after $24 \mathrm{~h}$ of treatment, the levels of other RC subunits reduced over the time. Of these tested proteins, the most sensitive to hexyl-TPP treatment were MT-CO1 and NDUFB8, while SDHB and CORE1 were less affected. The decrease of MTCO1 and NDUFB8 was statistically significant at 12 or $24 \mathrm{~h}$, respectively; however, there was already a strong decrease at $2 \mathrm{~h}$ (MTCO1) and 6h (NDUFB8) of treatment.

To investigate the effect of hexyl-TPP on the assembly of IMM proteins into their native respiratory chain complexes and supercomplexes ${ }^{23}$, we then performed Blue Native PAGE (BN-PAGE) analysis (Fig. $2 B, C)$. C2C12 cells were treated with $2 \mu \mathrm{M}$ hexyl-TPP at different timepoints, 2, 6, 12 and 24 hours. The effects of these treatments on complex IV (CIV) stability and formation were evaluated by immunodetection with the antibody recognising MT-CO1, after the native electrophoresis of samples solubilised with digitonin (Fig. 2B). We observed a decrease in the amounts of CIV bound to the supercomplexes after 6 hours of treatment, whereas the decrease of 'free' CIV was visible at 12 hours and more pronounced after 24 hours of treatment. The observed early effect on the supercomplexes formation could be due to specific disassembly or lack of assembly of CIV into these structures. Alternatively, it could be a consequence of earlier disruptions to other components of the respiratory chain supercomplexes. Therefore, we further performed BN-PAGE analysis followed by immunoblotting against NDUFB8 and we observed defects in assembly of complex I. Given that NDUFB8 is a $\mathrm{Cl}$ supernumerary subunit necessary for the assembly of the holocomplex ${ }^{24}$, this can explain why the levels of supercomplexes were already reduced at 6 hours of exposure to hexyl-TPP (Fig. 2B,C). Together, these results suggest that the hexyl-TPP treatment may impact newly synthetized or unassembled protein subunits.

A similar effect on steady-state protein levels was also observed in human HepG2 cells treated with hexyl-TPP, suggesting that this is not a cell-line specific phenomenon (Fig. S3B). To exclude the possibility of a decreased mitochondrial protein solubility induced by hexyl-TPP treatment, we also performed mild cellular lysis followed by SDS extraction of insoluble pelleted proteins (Fig. S3C). As MTCO1 protein remained detergent-soluble after the treatment with hexyl-TPP, we excluded the possibility that the observed decrease was simply due to protein aggregation.

We also tested the hypothesis that TPP treatment may affect the expression of genes coding for different subunits of complexes I and IV, whose steady state protein levels were the most affected by hexyl-TPP treatment (Fig. 2D). The nuclear encoded mRNA transcript levels of genes for CI (NDUFB8) or CIV (COX4/1) remained unchanged. However, while transcript levels of the mitochondrial encoded gene for CIV (MT-CO1) is unchanged, transcript levels of gene for $\mathrm{Cl}$ (MT-ND6) were increased. Therefore, this experiment ruled out the possibility of hexyl-TPP decreasing mtDNA transcription or downregulation of nuclear genes encoding respiratory chain components. 
bioRxiv preprint doi: https://doi.org/10.1101/2021.03.09.434520; this version posted May 18,2021 . The copyright holder for this preprint (which was not certified by peer review) is the author/funder, who has granted bioRxiv a license to display the preprint in perpetuity. It is made available under aCC-BY-NC-ND 4.0 International license.
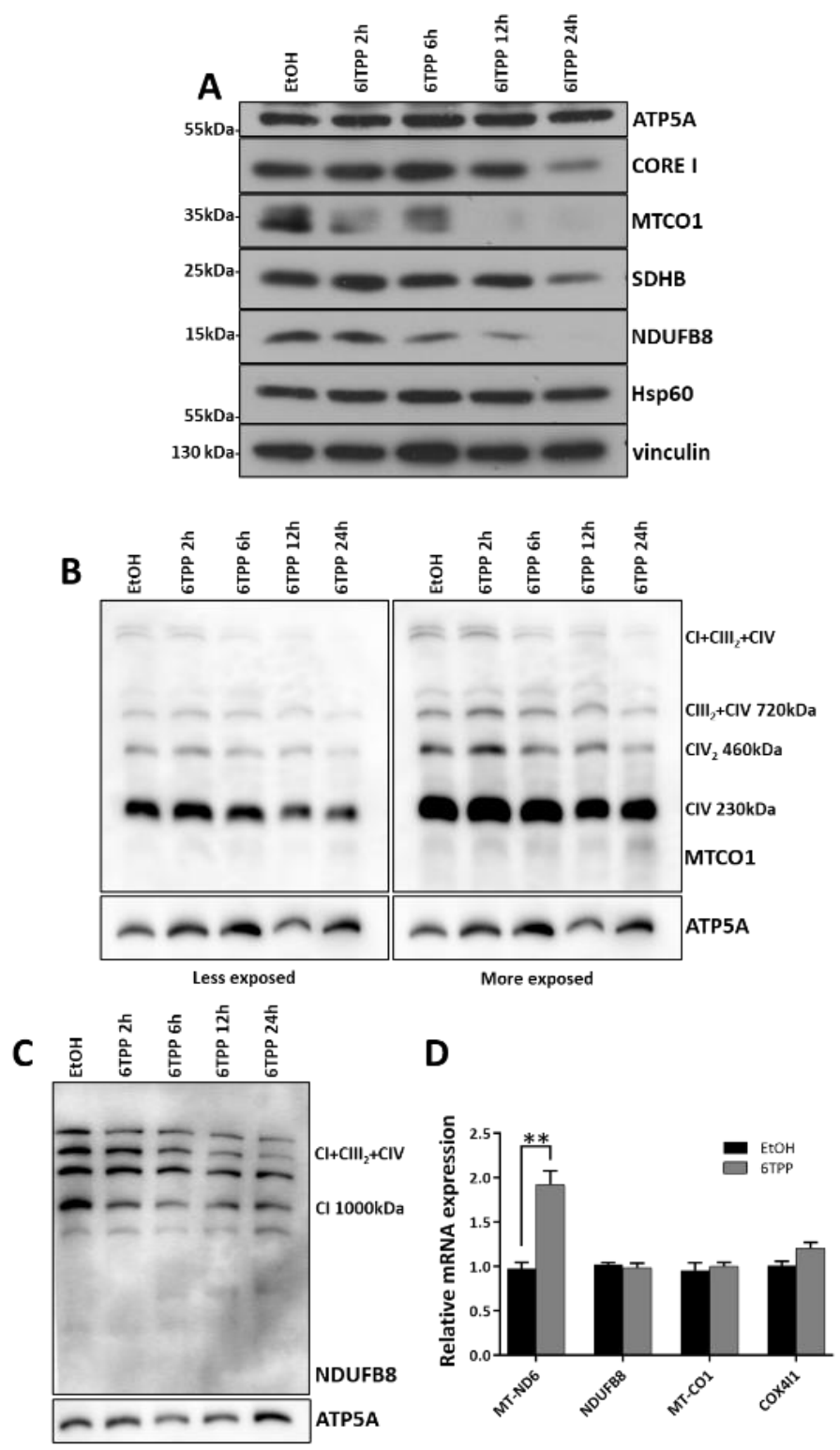

Figure 2. Treatment with hexyl-TPP leads to a decrease of OXPHOS complexes in a time dependent manner. A. Steady-state protein levels of respiratory chain components, NDUFB8 of complex I, SDHB of complex II, CORE I of complex III and MT-CO1 of complex IV and of complex V (ATP5A) in C2C12 cells treated with ethanol (EtOH) or with $2 \mu M$ hexyl-TPP over different timepoints as detected by immunodetection with specific antibodies against the indicated subunits. Vinculin and Hsp60 were used as loading controls. Scan of original immunoblot can be found in Fig. S7. B,C. 1D-BNGE and immunodetection analysis showing the decrease of fully assembled (B) complex IV, detected with anti-MT-CO1 and (C) complex I, detected with antiNDUFB8 after $2 \mu \mathrm{M}$ hexyl-TPP treatment at different timepoints, in C2C12 cells. D. Quantification of mRNA levels in C2C12 cells treated with ethanol or hexyl-TPP for 24 hours by RT-PCR normalized to actin MRNA and expressed relative to control cells. Results represent three independent experiments, error bars indicate mean \pm SEM, ${ }^{* *}$ indicate $p<0.01$ in unpaired student $t$-test.

\section{Hexyl-TPP treatment results in a decrease in MCUC steady state protein levels and enhanced OPA1 cleavage}

In order to elucidate whether the decrease in the levels of OXPHOS-related proteins upon exposure to hexyl-TPP was target-specific or due to a global reduction of mitochondrial proteins, the levels of OMM (TOM20, VDAC), matrix (GRP75 and citrate synthase), IMM (MIC60, MIC19, MCU) and IMS (MICU1, MICU2, cytochrome $c$ ) proteins were determined using immunoblot analyses (Figure 3a-d, S8A-C, S9A). 
We observed no changes in the levels of OMM and matrix proteins. Interestingly, whilst the levels of the mitochondrial calcium uniporter (MCU) and its associated gatekeepers (MICU1 and MICU2) were decreased (Fig. S4A), other steady state protein levels of IMM and IMS proteins such as MIC60, MIC19 and cytochrome $c$, remained unchanged after a 24-hour treatment.

We also analysed the protein level of the IMM GTPase OPA1, which is involved in the regulation of both mitochondrial IMM fusion and cristae maintenance ${ }^{25}$. In response to different stress stimuli including depolarizing agents such as FCCP or others, a transient depolarization of the IMM can lead to the activation of the metalloprotease OMA1, which cleaves the long OPA1 isoforms (L-OPA1) into shorter OPA1 (S-OPA1) isoforms ${ }^{252627}$. We therefore detected OPA1 isoforms after 24 hours exposure to $2 \mu \mathrm{M}$ hexyl-TPP and observed a reduction in L-OPA1 isoforms (L1 and L2), which was accompanied by the accumulation of the shorter OPA1 isoforms (S1 and S3) resulting from OPA1 cleavage (Fig. 3E). Collectively these results indicate that rather than a global, non-specific effect on the mitochondrial proteome, the effect of hexyl-TPP is selective for particular mitochondrial proteins and can induce a mitochondrial stress mediating OPA1-cleavage.
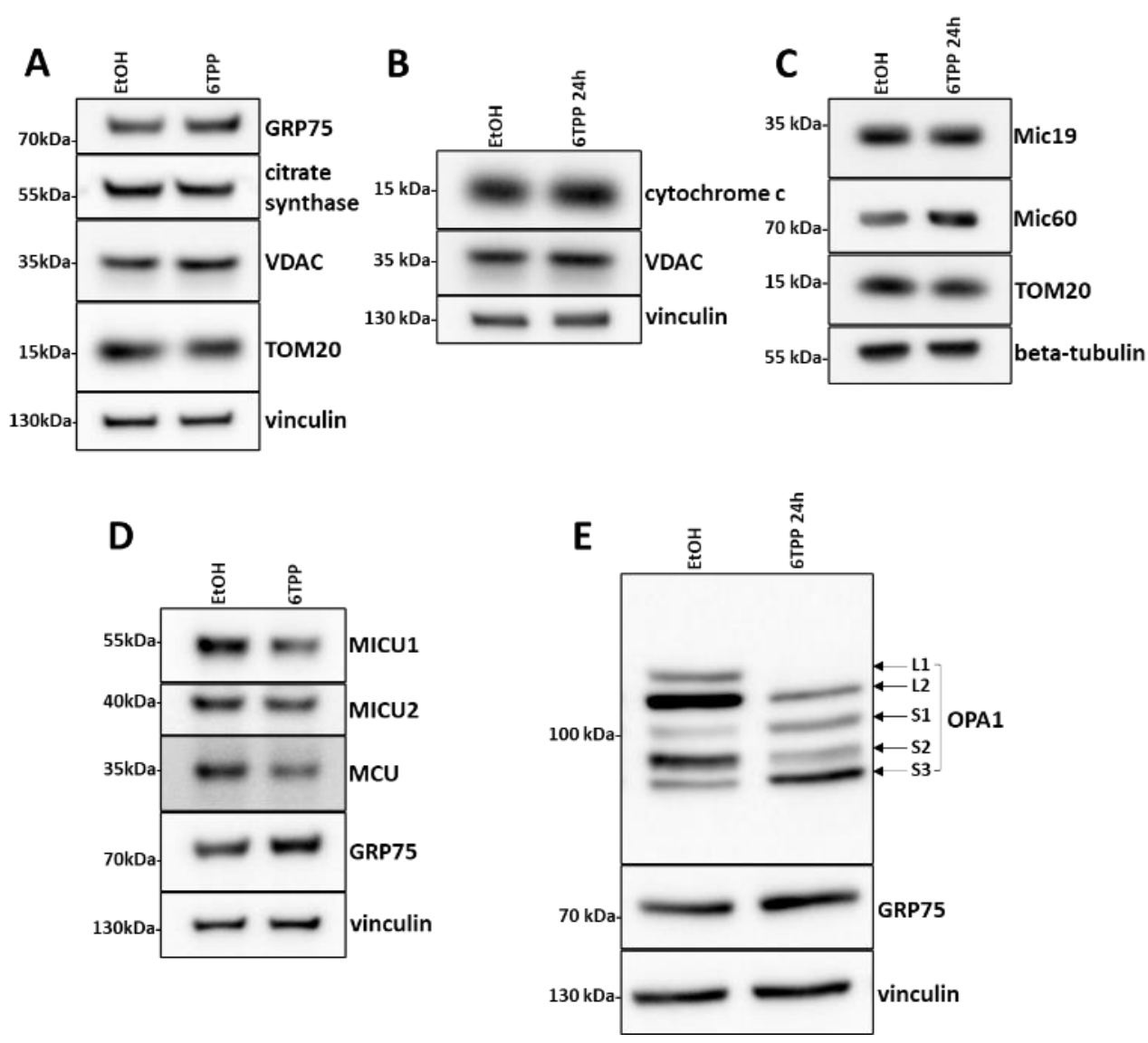

Figure 3. Treatment with hexyl-TPP leads to the decrease of MCUC subunits and OPA1 cleavage. A. Steady-state levels of $O M M$ and matrix proteins from C2C12 cells treated with ethanol or $2 \mu \mathrm{M}$ hexyl-TPP after $24 \mathrm{~h}$ treatment as detected by immunoblotting. Vinculin was used as a loading control. B-D. Steady-state levels of IMM and IMS proteins from C2C12 cells treated with ethanol or $2 \mu M$ hexyl-TPP after $24 \mathrm{~h}$ treatment. Vinculin or beta-tubulin were used as a cytoplasmic loading control and VDAC, TOM20 or GRP75 were used as a mitochondrial loading control. E. The effect of $2 \mu M$ hexyl-TPP treatment in C2C12 on OPA1 processing and stability. Vinculin and GRP75 were used as loading control. Scans of original immunoblots from A-E can be found in Fig. S8 A, B, C and S9 A, B, respectively.

\section{Hexyl-TPP treatment alters mitochondrial morphology}


The cleavage of IMM-inserted L-OPA1 and the resulting accumulation of short OPA1 isoforms have been associated with a mitochondrial fragmentation as well as a disorganised and swollen cristae structure $^{27}$. To investigate whether the accumulation of the short OPA1 isoforms after hexyl-TPP exposure results in a similar phenotype, we used confocal and electron microscopy to assess mitochondrial morphology and cristae ultrastructure. We observed that hexyl-TPP treatments leads to a remodelling of the mitochondrial network.

After 2- and 24-hour treatment with hexyl-TPP we did not observe increased mitochondrial fragmentation using confocal microscopy, instead mitochondria were aggregated close to the perinuclear area and were swollen with an increase in the mitochondrial area (Fig. 4A-D). This phenotype was confirmed by electron microscopy (Fig. 4E,F), where we observed not only rounded mitochondria but also an increase of the mitochondrial area. Electron microscopy also revealed a loss of the cristae structure (Fig. 4E).

Interestingly, after a shorter, one hour hexyl-TPP treatment we observed a significantly increased number of cells with fragmented mitochondria compared to the control. This phenotype was characterized by a reduction in the mitochondrial area, which was accompanied by an increase in mitochondrial number per unit area (Fig. S5A-D). These results suggest that short-term hexyl-TPP treatment induced first mitochondrial fragmentation, which shifted towards mitochondrial aggregation and swelling upon longer treatments.

To better characterise the changes in mitochondrial ultrastructure we further performed a 3D reconstruction of mitochondria in chemically fixed and cryo-sectioned cells. We confirmed the loss of cristae in mitochondria from cells exposed to $2 \mu \mathrm{M}$ of hexyl-TPP for 24 hours compared to control mitochondria. The negative staining of mitochondrial membrane enabled us to visualize the IMM, which remained unchanged after the treatment with hexyl-TPP and no rupture in IMM was detected (Fig. 5A-D). This phenotype, i.e. loss of cristae as well as mitochondrial fragmentation and swelling are often associated with cell death ${ }^{28}$. However, immunoblot analysis of caspase- 3 and PARP cleavage, two hallmarks of the apoptotic program ${ }^{293031}$, did not suggest apoptosis, even after a prolonged hexyl-TPP exposure (24 and 72 h) (Fig. S5E). 
bioRxiv preprint doi: https://doi.org/10.1101/2021.03.09.434520; this version posted May 18, 2021. The copyright holder for this preprint (which was not certified by peer review) is the author/funder, who has granted bioRxiv a license to display the preprint in perpetuity. It is made available under aCC-BY-NC-ND 4.0 International license.
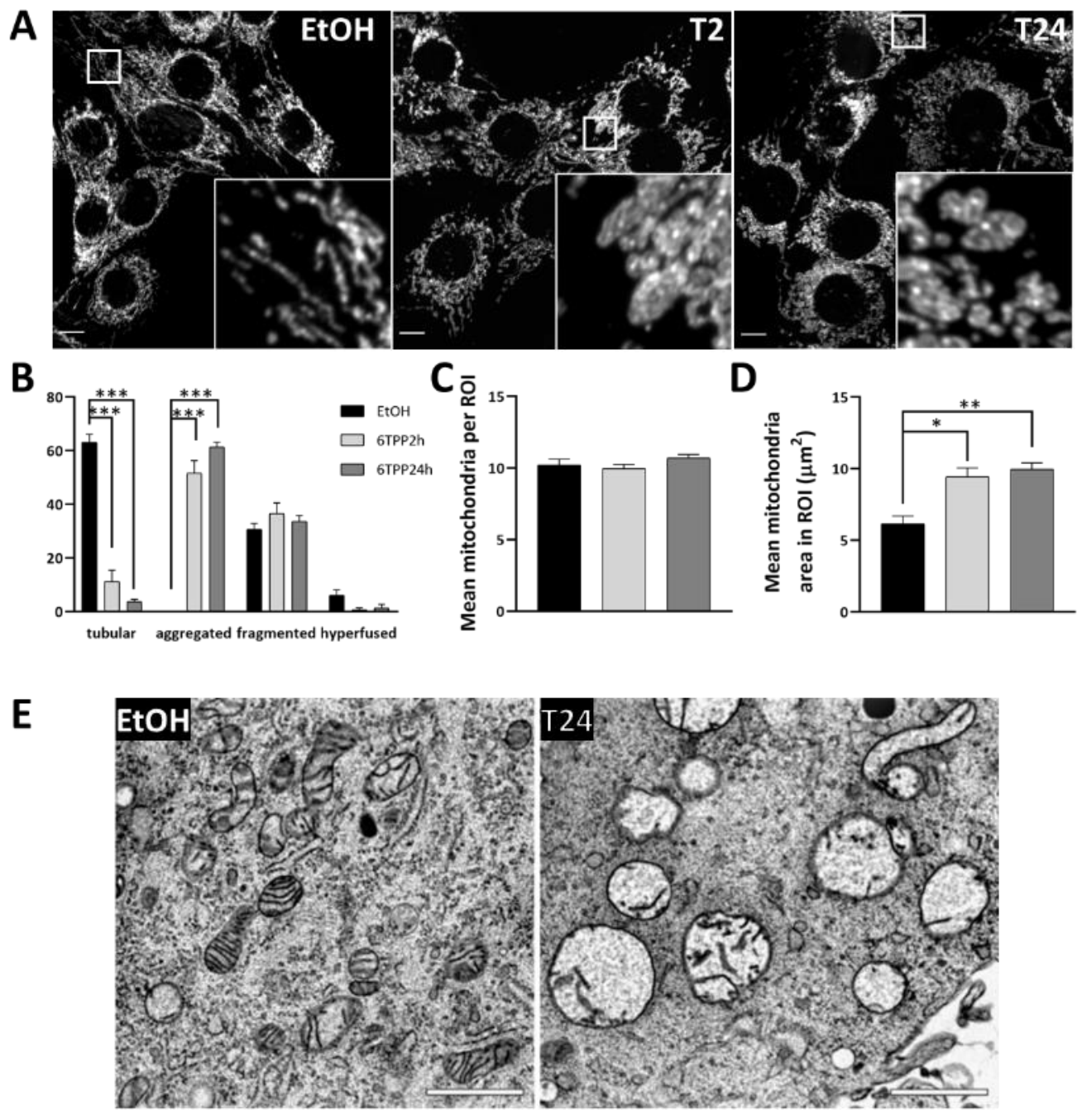

$\mathbf{F}$



Figure 4. Treatment with hexyl-TPP alters mitochondrial morphology. A. Representative images of mitochondrial morphology in C2C12 cells treated with ethanol or $2 \mu \mathrm{M}$ hexyl-TPP for 2 or 24 hours observed by confocal microscopy. Mitochondria were labelled using an anti-TOM20 antibody. Scale bars $10 \mu \mathrm{m}$. B. Quantification of mitochondrial morphology from (A). C. Mitochondrial morphology quantified for mean mitochondrial number for each region of interest (ROI). $D$. Mitochondrial morphology quantified for mean mitochondrial area per mitochondrion. All data are shown as mean \pm SEM, for $B$ at least 50 cells, for $C$ and $D 15$ cells for each experiment were quantified; three independent experiments were performed. For B two-way ANOVA and Tukey's multiple-comparisons test was used. For C and D ordinary one-way ANOVA and Tukey's multiple comparisons test was used. E. Representative images from transmission electron microscopy of mitochondrial ultrastructure in C2C12 cells treated with vehicle or $2 \mu \mathrm{M}$ hexyl-TPP for 24 hours and prepared by HPF/FS protocol. Scale bars $0.5 \mu \mathrm{m}$. F. Quantification of mitochondria area from E. The area of at least 250 mitochondria was quantified for both, control (EtOH) and treatment (6TPP). 

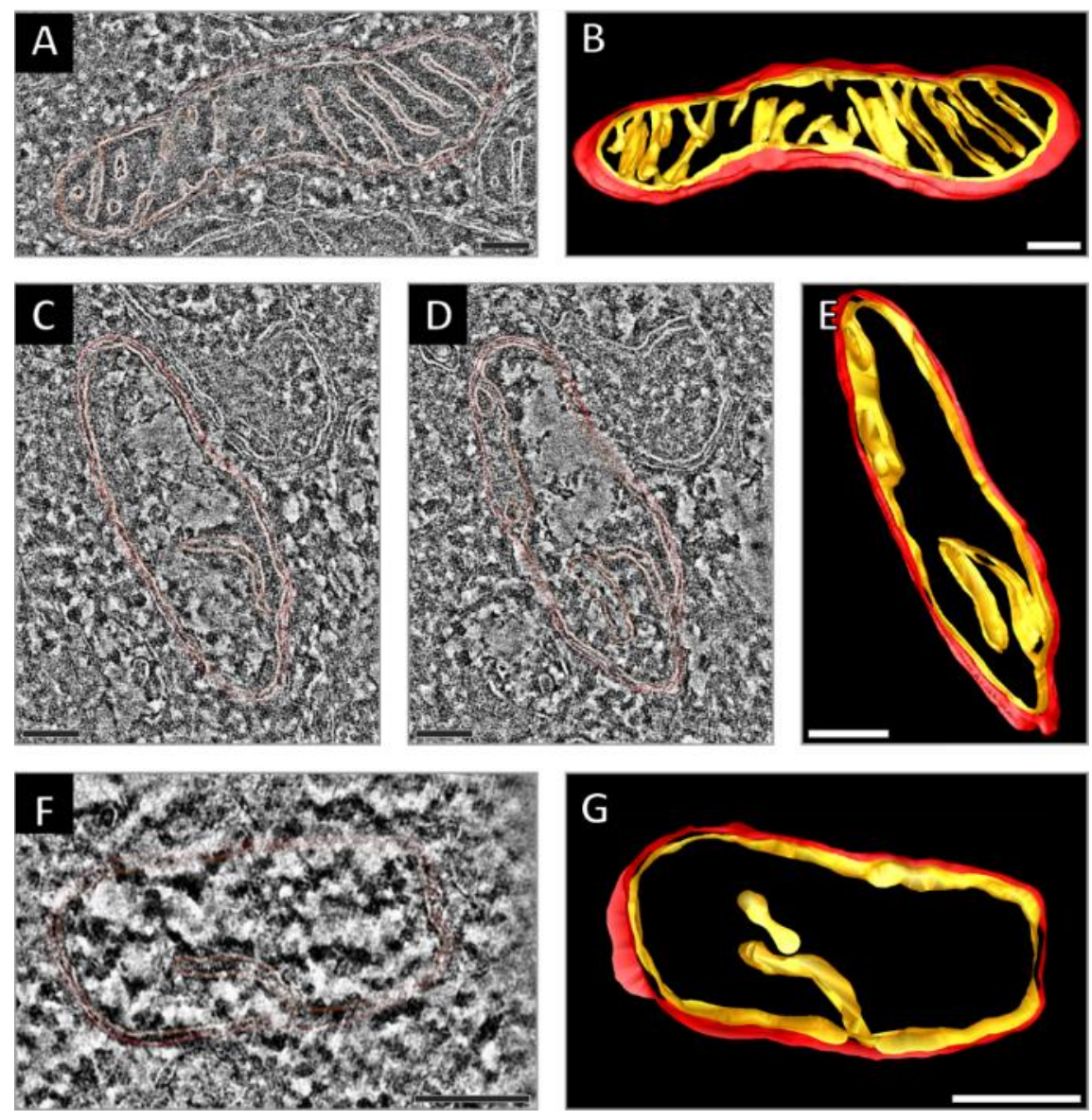

Figure 5. Hexyl-TPP treatment leads to cristae loss but does not impact inner boundary membrane. Representative images of tomographic slice and 3D reconstruction of mitochondria in control and hexyl-TPP treated cells, mitochondrial membranes are represented in red (outer) and orange (inner). A, B control C-G 24 hours exposure to $2 \mu M$ hexyl-TPP. The difference in height between $C$ and $D$ is $\sim 10 \mathrm{~nm}$. Each tomogram virtual slice represents thickness $0.56 \mathrm{~nm}$. Scale bars: $100 \mathrm{~nm}$.

\section{Loss of IMM proteins is membrane potential independent}

Acute exposure to TPP derivatives has been reported to dramatically decrease the mitochondrial membrane potential ${ }^{21}$. A 24-hour treatment with $2 \mu \mathrm{M}$ hexyl-TPP decreased the membrane potential to the same level as an equal concentration of FCCP, a strong uncoupler of oxidative phosphorylation (Fig. 6A). Since the drop of the membrane potential was proposed to cause an impairment of mitochondrial translation ${ }^{3233}$ and since it is crucial for the import of mitochondrial protein precursors from the cytosol ${ }^{34}$; we further hypothesised, that the drop of the membrane potential could indeed be the main trigger of decreased IMM protein levels. However, a 24-hour treatment with $2 \mu \mathrm{M} \mathrm{FCCP} \mathrm{did}$ not result in a decrease of selected steady-state RC protein levels, while a treatment with hexyl-TPP did, suggesting that a drop in membrane potential alone is not causative of the effect of hexyl-TPP (Fig. 6B). 
A

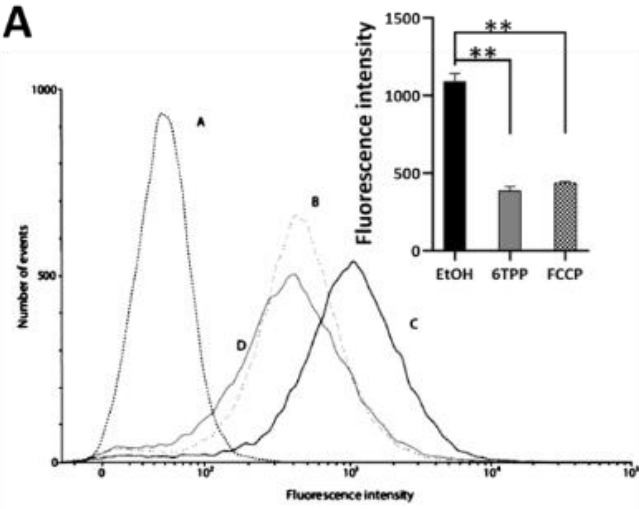

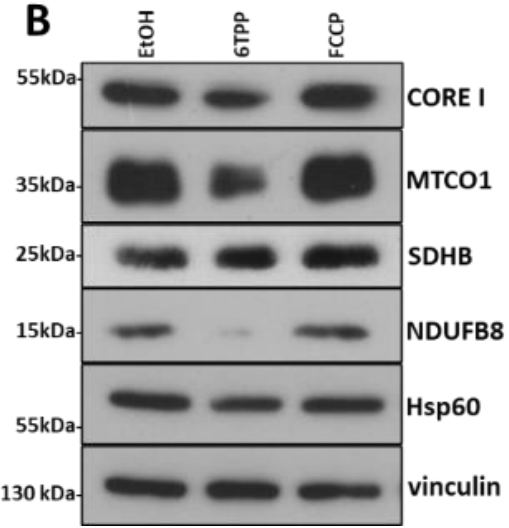

Figure 6. Drop in membrane potential alone is not causative of the effect of hexyl-TPP A. The effect of 24 hours treatment of $2 \mu \mathrm{M}$ hexyl-TPP or $2 \mu \mathrm{M}$ FCCP compared to control treated with vehicle on mitochondrial membrane potential in C2C12 cells. Cells were stained with $50 \mathrm{nM}$ TMRM, followed by flow cytometry analysis. A: unstained EtOH, B: $2 \mu M$ FCCP C: EtOH D: $2 \mu \mathrm{M}$ hexyl-TPP. The geometric mean fluorescence intensities are shown in a representative histogram. The average of three independent experiments, is shown in a bar chart, error bars indicate mean $\pm S E M,{ }^{* *}$ indicate $p<0.01$ in unpaired student $t$ test. B. Steady-state protein levels of different OxPhos subunits from C2C12 cells treated with vehicle/2 $\mu M$ FCCP/2 $\mu M$ hexylTPP for 24 hours as detected by immunoblotting. Scan of original immunoblot can be found in Fig. S10.

\section{The effects of hexyl-TPP on mitochondrial structure and function are reversible}

Previous studies have shown that TPP derivatives disrupt mitochondrial bioenergetics ${ }^{1921}$ and here we show that these functional changes are accompanied by changes in mitochondrial morphology as well as a selective disappearance of IMM proteins. We therefore investigated if both these effects could be reversed (and how quickly) when the toxic chemical was removed.

Cells were treated with hexyl-TPP for 24 hours, and then incubated in a fresh medium without TPP for 1 or 24 hours (Fig. 7A-D). In all the rescue experiments, the hexyl TPP-containing medium was replaced twice, in a 30 minute interval, to remove the TPP released from cells ${ }^{10}$. Just one hour after the removal of TPP we observed that mitochondria were less swollen, although the mitochondrial network was still fragmented. After 24 hours without TPP, cells completely recovered their mitochondrial network to a state comparable to control cells. The functional disruptions in mitochondrial respiration also returned to control levels after 24 hours without TPP (Fig. 7E).

Finally, we looked at mitochondrial protein levels after the removal of hexyl-TPP at 24-, 48- and 72hours. Whilst protein levels were still significantly reduced 24 hours after the removal of TPP, we observed a gradual increase in protein levels after 48 and 72 hours (Fig. 7F). Together, these data indicate that the effects of TPP derivatives on mitochondrial morphology, bioenergetics and IMM protein levels are reversible over time upon the removal of TPP. 



C

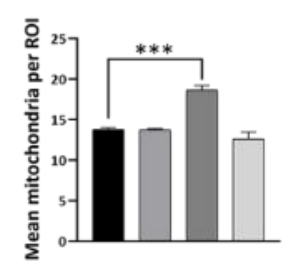

D



$\mathbf{E}$

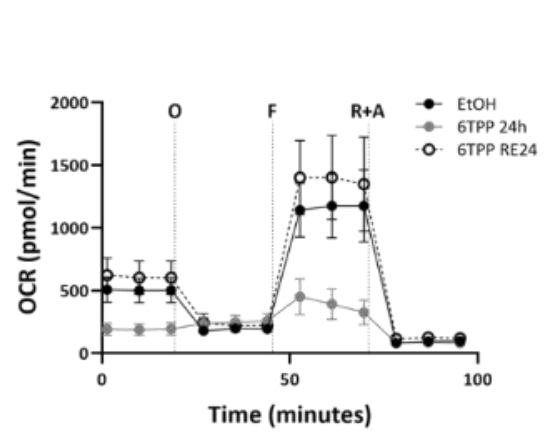

$\mathbf{F}$

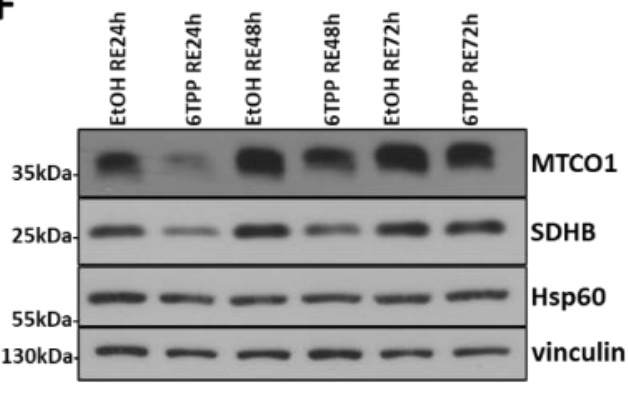

Figure 7 The effect of chronic treatment with hexyl-TPP is fully reversible. A. Representative images of mitochondrial morphology in C2C12 cells treated with vehicle (EtOH) or $2 \mu \mathrm{M}$ hexyl-TPP for 24 hours, followed by washes and incubation in cell growth medium without the treatment for 1 or 24 hours. Mitochondria were labelled using an anti-TOM20 antibody. Scale bars $10 \mu \mathrm{m}$. B. Quantification of mitochondrial morphology from A showing three independent experiments, for each at least 40 cells have been quantified, error bars are presented as mean \pm SEM, $n=3$, two-way ANOVA and Tukey's multiplecomparisons test was used. C. Mitochondrial morphology quantified for mean mitochondrial number for each region of interest (ROI). D. Mitochondrial morphology quantified for mean mitochondrial area per mitochondrion. All data for $C$ and $D$ are shown as mean \pm SEM, at least 15 cells for each experiment were quantified; three independent experiments were performed. Ordinary one-way ANOVA and Tukey's multiple comparisons test was used. E. Seahorse analysis of oxygen consumption rate in $\mathrm{C} 2 \mathrm{C} 12$ cells from control (EtOH) compared to hexyl-TPP treatment for 24 hours and 24 hours treatment with hexyl-TPP followed by 24-hour incubation in cell growth medium without the treatment. Cells were seeded in a Seahorse XF-24 plate and incubated overnight. Cellular media was replaced with XF Assay Media and allowed to be equilibrate for $1 \mathrm{~h}$ at $37^{\circ} \mathrm{C}, \mathrm{CO}_{2}$ free incubator. Basal respiration was measured, followed by sequential injections of $1 \mu \mathrm{M}$ oligomycin (O), $2 \mu \mathrm{M}$ $F C C P(F)$ and $1 \mu M$ rotenone $+1 \mu \mathrm{M}$ antimycin $(R+A)$. All data are mean $\pm S E M, n=3$. $F$. C2C12 cells were treated with vehicle or $2 \mu M$ hexyl-TPP for 24 hours, followed by washes and incubation in cell growth medium without the treatment for 24,48 and 72 hours, the steady state protein levels of rescue experiment were detected on immunoblot. Scan of original immunoblot can be found in Fig. S11.

\section{Similar effects of the TPP moiety can be observed in MitoQ but not in the less hydrophobic TPP- hexanol}

To understand if the effects described in this study were specific to alkyl-TPP derivatives or can be observed in other TPP compounds, we tested two different TPP derivatives, namely the well-known mitochondrially targeted antioxidant MitoQ and a hydrophilic analogue of hexyl-TPP, TPP-hexanol (Fig. S1D-E). A treatment with MitoQ led to a decrease in the steady state protein levels of IMM proteins 
even at a ten-fold lower concentration compared to hexyl-TPP (Fig. 8A). This result likely reflects the differences in overall hydrophobicity between the two molecules with MitoQ being more hydrophobic. On the contrary, the treatment with TPP-hexanol did not result in a decrease in the selected steady state protein levels even at concentrations as high as $100 \mu \mathrm{M}$ (Fig. 8B), most likely due to the substantially lower hydrophobicity caused by the hydroxyl group.
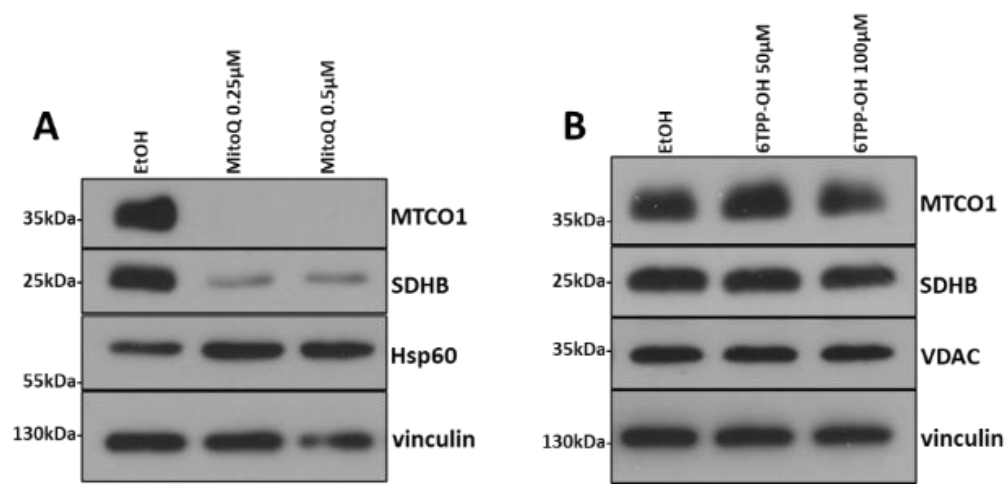

Figure $\mathbf{8} \mathbf{A}$. Immunoblotting for steady-state levels of respiratory chain complex IV (anti-MTCO1) and complex II (anti-SDHB) from C2C12 cells treated with vehicle or different concentration of MitoQ for 24h. B. Immunoblotting for steady-state levels of respiratory chain complex IV (anti-MTCO1) and complex II (anti-SDHB) from C2C12 cells treated with vehicle or different concentration of hexanol-TPP. Scans of original immunoblots from A and B can be found in Fig. S12 and S13, respectively.

\section{DISCUSSION:}

Lipophilic cations based on the triphenylphosphonium moiety used to target molecules into mitochondria are well known to associate with phospholipid biomembranes, particularly the inner mitochondrial membrane ${ }^{35}$ and have been shown to interact directly with cellular proteins, in particular $\mathrm{Hsp}^{30^{36}}$. We therefore hypothesised that in addition to disrupting mitochondrial bioenergetics they may also disrupt specific proteins, such as subunits of the respiratory chain complexes and/or cause a disruption of the assembly of its supercomplexes. In the experiments presented here we observed that exposure of cells to alkyl-TPP derivatives decreases the steady-state protein levels of different subunits of respiratory chain complexes (MTCO1, NDUFB8, SDHB, CORE I) in a concentration- and time-dependent manner (Fig. 1A-C, 2A, Fig. S2A- $\mathrm{C}$ and Fig. S3A) and also with an increasing potency as the hydrophobicity increases. Interestingly, the decrease of supercomplexes as well as steady-state protein levels was observed prior to the decrease in the abundance of 'free' complexes (Fig. 2A-C, Fig. S3A). This result suggests that the treatment with hexyl-TPP causes a rapid detrimental effect on newly synthesised or unassembled proteins, rather than a decrease of proteins as a result of interaction with the inner membrane complexes inducing their subsequent degradation. We also showed that earlier steps of mtDNA expression, i.e. mtDNA levels and transcription, are not negatively impacted by a TPP treatment (Fig. 2D).

When we examined the effect of hexyl-TPP treatment on the levels of a range of different mitochondrial proteins, we found out that the proteins of the OMM and matrix did not decrease in the treated cells (Fig. 3A), while some but not all of the IMM and intermembrane space proteins were affected (Fig. 3B-D). These results suggest that the mechanism of TPP action is rather selective in targeting proteins of the IMM. We observed a selective reduction in OXPHOS components and the MCU complex, which are responsible for mitochondrial respiration and the regulation of calcium entry into the mitochondrial matrix, respectively. This selective behaviour might be due to a possible link between these two functional systems. The levels of calcium inside mitochondria have been shown directly to impact the activity of the Krebs cycle enzymes ${ }^{37}$, activity of complex IV ${ }^{38}$ or ATP synthase ${ }^{39}$, and MCU itself directly interacts with ATP synthase ${ }^{40}$. The mitochondrial calcium uniporter regulator 1 
(MCUR1) has also been described as a scaffold protein for both $\mathrm{MCU}^{41}$ and cytochrome $c$ oxidase ${ }^{42}$. The observed decrease in MCUC protein levels therefore could also be a result of an impairment of OXPHOS complexes by the TPP compounds.

The mitochondrial membrane remodelling in response to stress, such as a drop of a membrane potential, is, in part, regulated by the IMM GTPase OPA1. Stress-induced OPA1 processing regulated by the IMM peptidase OMA1 causes an accumulation of its shorter forms, which results in mitochondrial fragmentation and cristae architecture remodelling ${ }^{26}$. The exposure to hexyl-TPP did indeed lead to OPA1 cleavage (Fig. 3E) suggesting that this regulator protein could be involved in the observed effects. This finding again suggests that TPP derivatives induce a selective stress response within the cell rather than causing non-specific disruptions.

Previous studies have shown that a drop in the mitochondrial membrane potential results in a disruption of mitochondrial translation ${ }^{3233}$ and that the membrane potential is crucial for the import of mitochondrial protein precursors from the cytosol ${ }^{34}$. Therefore, we hypothesized, that the drop of the mitochondrial membrane potential due to the treatment with hexyl-TPP (Fig. 6) might be a cause for the observed decrease in IMM protein levels. However, our results showed that an equal drop in membrane potential caused by $2 \mu \mathrm{M}$ of the uncoupler FCCP during equal times (Fig. 6A) did not lead to the same decrease in steady state protein levels (Fig. 6B). This rules out the possibility of the protein decrease being simply due to the dissipation of membrane potential and suggests a direct role for hexyl-TPP on mitochondrial protein synthesis and/or stability, by so far uncharacterised mechanisms.

As previously reported for the more hydrophobic dodecyl-TPP ${ }^{17}$, we observed that exposing cells to $2 \mu \mathrm{M}$ hexyl-TPP causes significant changes in mitochondrial morphology, including mitochondrial fragmentation and swelling (Fig. 4A-F, Fig. S5A-D). Using electron microscopy, we observed loss of cristae architecture but, perhaps surprisingly, electron tomography analysis did not find any ruptures of the IMM (Fig. 5A-G). Such an observation goes against the oft presented hypothesis that TPP derivatives behave as detergents virtually "dissolving" the IMM. The hydrophobicity of the TPP-linked molecules is clearly the most relevant physico-chemical parameter for their toxic effects. This phenomenon is evident since the more hydrophobic mitochondria-targeted antioxidant MitoQ exhibits stronger effects on IMM protein levels compared to the less hydrophobic congener of hexyl-TPP, TPP-hexanol (Fig. 8A,B).

Finally, we observed that alterations in the mitochondrial function and structure caused by hexyl-TPP treatment are fully reversible upon the removal of the molecule (Fig. 7A-F). This finding further strengthens the hypothesis that TPP-induced changes are the result of controlled processes, not due to non-specific toxicity.

The presented results are highly relevant both for the design of new mitochondrial probes and in the development of new mitochondria-targeted drugs. This study also provides a novel piece into the puzzle of interpreting the effect of TPP-conjugated molecules. Lastly, the specific effect of lipophilic TPP-based cations hints at a currently unknown process linking the disruption of the IMM to the changes in membrane protein levels, which warrants further study.

Our experiments looked at the effects of the TPP moiety after a relatively long in vitro exposure. In vivo, it is conceivable that the excretion and redistribution of the compound among organs and tissues would result in a fluctuation between the disrupted and recovery phenotypes we described in this study. On the other hand, in certain cells/tissues or under certain circumstances this might not be the case and these in vitro effects might be observed also in vivo; e.g. in the kidney, where the compounds may be concentrated over time, due to being the main route of excretion ${ }^{43}$. Importantly, there are already first mentions of the negative effects of TPP treatment in different tissues in vivo models ${ }^{44}$. 
We can conclude here that the TPP moiety used for the synthesis of a range of mitochondria-targeted compounds is not inert but initiates profound, specific and reversible effects on the mitochondrial structure and function, which must be taken into account whenever these compounds are used.

\section{METHODS}

\section{Materials}

Propyltriphenylphosphonium bromide (131563) and methyltriphenylphosphonium bromide (130079) were purchased from Sigma-Aldrich. Hexyltriphenylphoshonium bromide was obtained from Merck (842007). Actinomycin D (A1410), FCCP (C2920), oligomycin (75351), rotenone (R8875) and antimycin A (A8674) were obtained from Sigma-Aldrich. MitoQ and TPP-hexanol were kindly provided by Dr. Michael P. Murphy's lab (MRC Mitochondrial Biology Unit, Cambridge, UK).

Antibodies

Rabbit monoclonal anti-TOM20 (ab232589), mouse monoclonal anti-MTCO1 (ab14705), anti-ATP5a (ab14748), anti-VDAC1 (ab14734), anti-SDHB (ab14714), anti-NDFUB8 (ab110242), anti-ubiquinolcytochrome c reductase core protein I (ab110252), anti-GRP75 (ab2799), anti-MIC60 (ab110329), anticytochrome c (ab110325), rabbit polyclonal anti-citrate synthase (ab96600) and anti-MICU2 (ab101465) were purchased from Abcam. Antibodies mouse monoclonal anti-Hsp60 (sc136291) and anti-alpha-tubulin (sc23948) were obtained from Santa-Cruz Biotechnology. Antibodies rabbit polyclonal anti-PARP (\#9542), anti-cleaved caspase 3 (\#9661) and rabbit monoclonal antiMICU1(\#12524) were purchased from Cell Signalling Technology. Antibodies mouse monoclonal antivinculin (V4505), anti-OPA1 (612607), rabbit polyclonal anti-MIC19 (25625-1-AP) and rabbit monoclonal anti-MCU (14997) were purchased from Sigma-Aldrich, BD Transduction Laboratories Proteintech and BioTech, respectively.

\section{Cell lines, culture conditions and cell proliferation assay}

C2C12 mouse myoblasts cells, and HepG2 human liver cancer cells were obtained from American Type Culture Collection (ATCC). Cells were cultured in low-glucose (1 g/l) Dulbecco's Modified Eagle's medium (DMEM) medium supplemented with $10 \%$ fetal bovine serum (FBS) and $0.5 \mathrm{mM}$ L-glutamine in humidified atmosphere at $37^{\circ} \mathrm{C}$ and $5 \% \mathrm{CO} 2$ (all from Gibco-Life Technologies).

TPP compounds were dissolved in ethanol, Actinomycin D, FCCP, oligomycin, rotenone and antimycin A were dissolved in DMSO, final concentration of vehicle used for any experiment was below $0.5 \%$.

\section{Denaturing and native electrophoresis, immunoblot, and immunodetection}

Samples for blue-native gel electrophoresis (BNGE) were prepared as described previously ${ }^{45}$. For the solubilisation, $2 \%$ digitonin was used. Samples were run through pre-cast Native PAGE Novex 3-12 \% Bis-Tris Protein Gels (Thermo Fisher Scientific) according to the manufacturer's instructions.

Samples for immunoblot were extracted in $1.5 \%$ DDM lysis buffer (D4641, Sigma-Aldrich) or RIPA lysis buffer (R0278, Sigma-Aldrich). $10 \mu \mathrm{g}$ aliquots of total protein were transferred to hand-cast polyacrylamide gel or to Novex NuPAGE 4-12 \% Bis-Tris Precast Gels (Thermo Fisher Scientific).

Proteins were then wet-transferred to PVDF or nitrocellulose membranes, blocked in $10 \%$ non-fat milk (Marvel) in $0.1 \%$ Phosphate buffer saline (PBS)-Tween and incubated with specific primary antibodies overnight. The following day the membranes were washed in $0.1 \%$ PBS-Tween, incubated with Goat anti-Mouse IgG (W402B, Promega) or Goat anti-Rabbit IgG (W401B, Promega) secondary antibodies for 1 hour (h), and washed again in $0.1 \%$ PBS-Tween. Immunoreactive bands were visualized with ECL 
Western Blotting Detection Reagents (ThermoFisher) or Amesham ECL Prime Western Blotting Detection Reagent (GE Healthcare) and revealed manually on X-Ray films (Fuji; FOMA LP-T and FOMAFIX) or using a digital Amersham Imager 680 (GE Healthcare).

\section{Mitochondrial protein solubilisation}

$50,000 \mathrm{C} 2 \mathrm{C} 12$ cells were seeded in a 6 -well plate and cultured overnight. Cells were treated with $2 \mu \mathrm{M}$ hexyl-TPP or vehicle for $24 \mathrm{~h}$ before harvesting. Equal numbers of cells were pelleted and incubated in a mild lysis buffer ( $1 \%$ Triton-X, $150 \mathrm{mM} \mathrm{KCl}, 30 \mathrm{mM}$ Tris $\mathrm{pH} 7.5$ ) for 10 minutes (min) at room temperature (RT), followed by centrifugation at $8,000 \times g$ for 5 min at $4{ }^{\circ} \mathrm{C}$. Supernatants with soluble protein fractions were kept and pellets were washed once in PBS and centrifuged at 8,000 $\mathrm{g} g$ for 5 min at $4{ }^{\circ} \mathrm{C}$. Pellets were resolubilized by vortexing in $2 x$ Laemmli sample buffer and homogenized by passing through silica-based spin column. Soluble and insoluble fractions were analysed for the presence of selected proteins by immunoblot analysis.

\section{Gene expression analysis}

Total RNA extraction was performed using RNeasy Mini Kit (Qiagen, Germany) according to manufacturer's instructions. RT-PCR and CDNA transcription were carried out using PowerUp SYBR Green Master Mix (Applied Biosystems, USA) and gene-specific primers: mtco1: 5gacttgcaaccctacacgga-3, 5-gatggcgaagtgggcttttg-3; actb: 5-cctgaaccctaaggccaacc-3, 5atggcgtgagggagagcatag-3; coxIV: 5-gctccgtcttggtcttccg-3，5-cttcacaacactcccatgtgct-3; nd6: 5tattgccgctaccccaatcc-3, 5-gggggatgttggttgtgtttg-3; ndufb8: 5-tggggtgaaccgatacactg-3, 5agcacgtagagggaaggaca-3; atp5a1: 5-tttgcgctcggccattttg-3, 5-catctcagctgtgccagtcttc-3. Data were analysed using the comparative $\Delta \Delta \mathrm{Ct}$ method with values normalized to $\beta$-actin levels. Specificity of PCR reaction for each amplicon was tested by melting curve analysis.

\section{Flow cytometry}

Changes in the mitochondrial membrane potential were determined as the changes in tetramethylrhodamine methyl ester (TMRM, T668, Thermo Fisher Scientific) fluorescence according to the manufacturer's instructions with few modifications. Briefly, cells were treated with $2 \mu \mathrm{M}$ hexylTPP, $2 \mu \mathrm{M}$ FCCP or vehicle for $24 \mathrm{~h}$. Then, cells were washed with PBS and incubated in staining solution (50 nM TMRM in Hank's Balanced Salt Solution with $2 \mu \mathrm{M}$ vehicle/hexyl-TPP/FCCP) at $37^{\circ} \mathrm{C}$ for $30 \mathrm{~min}$. Finally, cells were harvested, centrifuged at $350 \times g$ for 3 min and resuspended in Hank's Balanced Salt Solution with 3\% FBS. Cells were then analysed using a BD Verse flow cytometer. 10, 000 events per sample were used and TMRM fluorescence was measured using the PE channel, with TMRM excitation at $488 \mathrm{~nm}$ and emission at $586 \mathrm{~nm}$.

\section{Immunofluorescence}

10,000 C2C12 cells were seeded on a glass coverslip in 24-well plates and incubated overnight. Cells were then treated with $2 \mu \mathrm{M}$ hexyl-TPP for different timepoints. The following steps were performed as previously described ${ }^{46}$. Briefly, the medium was removed, and cells were fixed with $5 \%$ paraformaldehyde at $37^{\circ} \mathrm{C}$ for $15 \mathrm{~min}$, washed in PBS, followed by quenching with $50 \mathrm{mM}$ ammonium chloride in PBS, washed with PBS and permeabilized with $0.1 \%$ Triton X-100 in PBS for 10 min. Next, cells were washed with PBS and blocked with $10 \%$ FBS in PBS. The cells on the coverslips were then incubated with the primary antibody (anti-TOM20) in $5 \%$ FBS in PBS for $2 \mathrm{~h}$ at RT. After three washes with $5 \%$ FBS in PBS, cells were incubated with secondary antibodies (Alexa Fluor 594, A11012, Thermofisher Scientific) in $5 \%$ FBS in PBS for $1 \mathrm{~h} \mathrm{RT}$, washed three times with PBS and mounted onto slides using fluorescence mounting medium (Dako). 


\section{Confocal imaging}

Fixed cells were imaged using 100X objective lens (NA1.4) on a Nikon Eclipse TiE inverted microscope with appropriate lasers using an Andor Dragonfly 500 confocal spinning disk system, equipped with a Zyla 4.2 PLUS sCMOS camera coupled with Fusion software (Andor). 7 stacks of $0.2 \mu \mathrm{m}$ each were acquired. Images were compiled by "max projection" using Fiji software ${ }^{47}$ and cells were classified as fragmented, tubular and hyperfused, similarly as previously described. ${ }^{48}$ In addition, we included aggregated mitochondria as a category of the mitochondrial morphology phenotype. For mitochondrial area and number analysis, $225 \mu \mathrm{m}^{2}$ of region of interest (ROI) was selected from max projection images and further analysed using the "Analyse particles" plugin in Fiji with a minimum area of $0.1 \mu \mathrm{m}^{2}$ as described previously ${ }^{48}$. Three independent experiments were performed. The numbers of cells quantified per conditions are indicated in figure legends, specifically for each experiment.

\section{Transmission electron microscopy}

\section{$\underline{\text { High-pressure freezing/freeze substitution (HPF/FS) }}$}

After $24 \mathrm{~h}$ of treatment with $2 \mu \mathrm{M}$ hexyl-TPP or vehicle, C2C12 cells were gently scraped and centrifuged at $350 \times \mathrm{g}$ for $5 \mathrm{~min}$. Cells were frozen using a Leica EM ICE high-pressure freezer in the presence of $20 \%$ bovine serum albumin $(\mathrm{w} / \mathrm{v})$. Frozen cells were cryo-substituted in medium containing $1 \%$ osmium tetroxide in $100 \%$ acetone at $-90{ }^{\circ} \mathrm{C}$ for $96 \mathrm{~h}$. Then, temperature was raised ( 5 $\left.{ }^{\circ} \mathrm{C} / 14 \mathrm{~h}\right)$ to $-20^{\circ} \mathrm{C}$ and after $24 \mathrm{~h}$ to $4{ }^{\circ} \mathrm{C}\left(3^{\circ} \mathrm{C} / 18 \mathrm{~h}\right)$. At RT, specimens were washed three times in 100 $\%$ acetone and infiltrated with $25 \%, 50 \%, 75 \%$ solutions of Embed 812 resin (EMS) in acetone for 1 h. Specimens were incubated in $100 \%$ resin overnight and finally embedded and polymerized at $60{ }^{\circ} \mathrm{C}$ for $48 \mathrm{~h}$. Ultrathin sections were counterstained in saturated ethanolic uranyl acetate and lead citrate and observed using TEM JEOL 1010 working at $80 \mathrm{kV}$.

\section{$\underline{\text { Tokuyasu cryosectioning }}$}

After $24 \mathrm{~h}$ of treatment with $2 \mu \mathrm{M}$ hexyl-TPP or vehicle, C2C12 were fixed in $4 \%$ formaldehyde with $2.5 \%$ glutaraldehyde in $0.1 \mathrm{M} \mathrm{HEPES}$. After $1 \mathrm{~h}$ incubation at RT and at $4{ }^{\circ} \mathrm{C}$ overnight, cells were washed with $0.1 \mathrm{M} \mathrm{HEPES}$ and cryoprotected in $2.3 \mathrm{M}$ sucrose for $72 \mathrm{~h}$ at $4{ }^{\circ} \mathrm{C}$ and frozen by plunging into liquid nitrogen. Ultrathin sections $(100 \mathrm{~nm})$ were cut at $-100{ }^{\circ} \mathrm{C}$, picked-up with $1.15 \mathrm{M}$ sucrose/ 1 $\%$ methylcellulose solution ( $25 \mathrm{cp}$, Sigma). Sections were incubated with gold nanoparticles $(15 \mathrm{~nm})$ for $3 \mathrm{~min}$, washed in $\mathrm{dH} 2 \mathrm{O}$, and contrasted/embedded using a mixture of $2 \%$ methylcellulose and $3 \%$ aq. uranyl acetate solution (9:1) for $10 \mathrm{~min}$. Samples were observed with a MegaView III camera mounted on a JEOL 1010 TEM. Electron tomography was done on a $200 \mathrm{kV}$ TEM JEOL 2100F equipped with motorized tilt stage and a Gatan K2-summit camera.

\section{Electron tomography}

SerialEM ${ }^{49}$ was used to automated acquisition of tilt series according to Saxton ${ }^{50}$ scheme in range $\pm 70^{\circ}$ with 131 projections. The tomogram reconstruction and model creation were done in ETomo software package $^{51}$. Reconstructed tomograms with isotropic pixel size $0.56 \mathrm{~nm}$ were filtered using low pass Fourier filter in Fiji software package ${ }^{47}$.

\section{Oxygen consumption measurement}

C2C12 cells treated with vehicle or $2 \mu \mathrm{M}$ hexyl-TPP were seeded overnight in 24-well culture plate at 30,000 cells/well. Next day, culture medium was removed and replaced with XF Assay Media from Seahorse XF DMEM medium supplemented with $5.5 \mathrm{mM}$ glucose, $1 \mathrm{mM}$ pyruvate, $4 \mathrm{mM}$ glutamate and $2 \mu \mathrm{M}$ hexyl-TPP/vehicle and incubated at $37^{\circ} \mathrm{C}$ for $1 \mathrm{~h}$. Oxygen consumption rate (OCR) was 
measured using the Seahorse XF24 Extracellular Analyzer. The assay consisted of three basal rate measurements, followed by sequential injections of $1 \mu \mathrm{M}$ oligomycin, $2 \mu \mathrm{M} \mathrm{FCCP}$ and $1 \mu \mathrm{M}$ rotenone $+1 \mu \mathrm{M}$ antimycin. Three rate measurements were performed after each injection. OCR was normalized to protein by the Bradford assay. The results are expressed as the average of three independent experiments.

\section{Statistics}

Graphs show mean \pm standard error of mean (SEM) from three independent experiments. Statistical significance was assessed using one-way ANOVA or two-way ANOVA with Tukey's multiple comparison or unpaired t-test in GraphPad Prism software, ${ }^{*} p<0.05,{ }^{* *} p<0.01,{ }^{* * *} p<0.001$.

\section{DATA AVAILABILITY STATMENT:}

All data are provided in the main article and in the supplementary. Raw data from the current study are available from the corresponding author on request.

\section{REFERENCES:}

1. Murphy, M. P. \& Hartley, R. C. Mitochondria as a therapeutic target for common pathologies. Nat. Rev. 17, 865-886 (2018).

2. Giacomello, M., Pyakurel, A., Glytsou, C. \& Scorrano, L. The cell biology of mitochondrial membrane dynamics. Nat. Rev. Mol. Cell Biol. 21, (2020).

3. Anderson, A. J., Jackson, T. D., Stroud, D. A. \& Stojanovski, D. Mitochondria - hubs for regulating cellular biochemistry : emerging concepts and networks. Open Bilogy 9, (2019).

4. Palty, R. et al. NCLX is an essential component of mitochondrial $\mathrm{Na} / \mathrm{Ca}$ exchange. PNAS 107, 436-441 (2010).

5. Fernández-Vizarra, E., Tiranti, V. \& Zeviani, M. Assembly of the oxidative phosphorylation system in humans : What we have learned by studying its defects. BBA - Mol. Cell Res. 1793, 200-211 (2009).

6. Krämer, S. D., Lombardi, D., Primorac, A., Thomae, A. V \& Wunderli-allenspach, H. LipidBilayer Permeation of Drug-Like Compounds. Chem. Biodivers. 6, 1900-1916 (2009).

7. Smith, R. A. J., Hartley, R. C., Cochemé, H. M. \& Murphy, M. P. Mitochondrial pharmacology. Trends Pharmacol. Sci. 33, 341-352 (2012).

8. Ross, M. F. et al. Liphophilic Triphenylphosphonium Cations as Tools in Mitochondrial Bioenergetics and Free Radical Biology. Biochem. 70, 222-230 (2005).

9. Murphy, M. P. Selective targeting of bioactive compounds to mitochondria. Trends Biotechnol. 7799, 326-330 (1997).

10. Ross, M. F. et al. Rapid and extensive uptake and activation of hydrophobic triphenylphosphonium cations within cells. Biochem. J. 411, 633-645 (2008).

11. Rohlenova, Katerina, Sachaphibulkij, K. et al. Selective Disruption of Respiratory Supercomplexes as a New Strategy to Suppress Her2 high Breast Cancer. Antioxid. Redox Signal. 26, 84-103 (2016).

12. Marrache, S., Pathak, R. K. \& Dhar, S. Detouring of cisplatin to access mitochondrial genome for overcoming resistance. Proc. Natl. Acad. Sci. 111, 10444-10449 (2014).

13. Liu, H. et al. Delivery of mitochondriotropic doxorubicin derivatives using self-assembling hyaluronic acid nanocarriers in doxorubicin-resistant breast cancer. Acta Pharmacol. Sin. 39, 1681-1692 (2018).

14. Biswas, S., Dodwadkar, N. S., Deshpande, P. P. \& Torchilin, V. P. Liposomes loaded with paclitaxel and modified with novel triphenylphosphonium-PEG-PE conjugate possess low toxicity, target mitochondria and demonstrate enhanced antitumor effects in vitro and in vivo. J. Control. Release 159, 393-402 (2012).

15. Cheng, G. et al. Mitochondria-targeted analogs of metformin exhibit enhanced 
antiproliferative and radiosensitizing effects in pancreatic cancer cells. Cancer Res. 263, 219227 (2017).

16. Manetta, A. et al. Novel phosphonium salts display in vitro and in vivo cytotoxic activity against human ovarian cancer cell lines. Gynecol. Oncol. 60, 203-212 (1996).

17. Gottwald, E. M. et al. The targeted anti-oxidant MitoQ causes mitochondrial swelling and depolarization in kidney tissue. Physiol. Rep. 6, 1-9 (2018).

18. Leo, S., Szabadkaia, G. \& Rizzuto, R. The Mitochondrial Antioxidants MitoE2 and MitoQ10 Increase Mitochondrial Ca2+ Load upon Cell Stimulation by Inhibiting Ca2+ Efflux from the Organelle. Ann N Y Acad Sci 1147, 264-274 (2008).

19. Reily, C. et al. Mitochondrially targeted compounds and their impact on cellular bioenergetics. Redox Biol. 1, 86-93 (2013).

20. U.S. National Library of Medicine. https://clinicaltrials.gov/ct2/show/NCT02364648?te

21. Trnka, J., Elkalaf, M. \& Anděl, M. Lipophilic triphenylphosphonium cations inhibit mitochondrial electron transport chain and induce mitochondrial proton leak. PLoS One 10, 114 (2015).

22. Elkalaf, M., Tuma, P., Weiszenstein, M., Polák, J. \& Trnka, J. Mitochondrial Probe Methyltriphenylphosphonium (TPMP) Inhibits the Krebs Cycle Enzyme 2- Oxoglutarate Dehydrogenase. PLoS One 11, 1-16 (2016).

23. Wittig, I., Braun, H. \& Scha, H. Blue native PAGE. Nat. Protoc. 1, 418-428 (2006).

24. Stroud, D. A. et al. Accessory subunits are integral for assembly and function of human mitochondrial complex I. Nat. Publ. Gr. 538, 123-126 (2016).

25. Macvicar, T. \& Langer, T. OPA1 processing in cell death and disease - the long and short of it. J. Cell Sci. 129, 2297-2306 (2016).

26. Ishihara, N., Fujita, Y., Oka, T. \& Mihara, K. Regulation of mitochondrial morphology through proteolytic cleavage of OPA1. EMBO 25, 2966-2977 (2006).

27. Anand, R. et al. -AAA protease YME1L and OMA1 cleave OPA1 to balance mitochondrial fusion and fission. JCB 204, 919-929 (2013).

28. Zemirli, N., Morel, E. \& Molino, D. Mitochondrial Dynamics in Basal and Stressful Conditions. Int. J. Mol. Sci. 1, 1-19 (2018).

29. Porter, A. G. \& Ja, R. U. Emerging roles of caspase-3 in apoptosis. Cell Death Differ. 6, 99-104 (1999).

30. Chiarugi, A. \& Moskowitz, M. A. PARP-1 - a prepetrator of apoptotic cell death? Science (80-. ). 297, 200-202 (2002).

31. Valero, J. G. et al. Bax-derived membrane-active peptides act as potent and direct inducers of apoptosis in cancer cells. J. Cell Sci. 124, 556-564 (2011).

32. Cote, C., Boulet, D. \& Poirier, J. Expression of the Mammalian Mitochondrial Genome Role for membrane potential in the production of mature translation products. J. Biol. Chem. 265, 7532-7538 (1990).

33. Cote, C., Poirier, J. \& Boulet, D. Expression of the Mammalian Mitochondrial Genome Stability of mitochondrial translation products as a function of membrane potential. J. Biol. Chem. 264, 8487-8490 (1989).

34. Martin, J., Mahlke, K. \& Pfanners, N. Role of an energized inner membrane in mitochondrial protein import. Delta psi drives the movement of presequences. J. Biol. Chem. 266, 1805118057 (1991).

35. Finichiu, P. G., James, A. M., Larsen, L., Smith, R. A. J. \& Murphy, M. P. Mitochondrial accumulation of a lipophilic cation conjugated to an ionisable group depends on membrane potential , $\mathrm{pH}$ gradient and $\mathrm{p} \mathrm{K} \mathrm{a}$ : implications for the design of mitochondrial probes and therapies. J Bioerg Biomembr 45, 165-173 (2013).

36. Leu, J. I., Zhang, P., Murphy, M. E., Marmorstein, R. \& George, D. L. Structural Basis for the Inhibition of HSP70 and DnaK Chaperones by Small-Molecule Targeting of a C - Terminal Allosteric Pocket. ACS Chem. Biol. 9, 2508-2516 (2014).

37. Wan, B., LaNoue, K. F., Cheung, J. Y. \& Scaduto, R. C. Regulation of citric acid cycle by calcium. 
J. Biol. Chem. 264, 13430-13439 (1989).

38. Vygodina, T., Kirichenko, A. \& Konstantinov, A. A. Direct Regulation of Cytochrome c Oxidase by Calcium lons. PLoS One 8, 1-11 (2013).

39. Territo, P. R., Mootha, V. K., French, S. A. \& Balaban, R. S. Ca2+ activation of heart mitochondrail oxidative phosphorylation : role of the F0/ F1-ATPase. Am. J. Physiol. Cell Physiol. 278, 423-435 (2020).

40. Huang, G. \& Docampo, R. The Mitochondrial Calcium Uniporter Interacts with Subunit c of the ATP Synthase of Trypanosomes and Humans. Mol. Biol. Physiol. 11, 1-16 (2020).

41. Tomar, D. et al. MCUR1 is a Scaffold Factor for the MCU Complex Function and Promotes Mitochondrial Bioergetics. Cell Rep. 15, 1673-1685 (2016).

42. Paupe, V., Prudent, J., Dassa, E. P., Rendon, O. Z. \& Shoubridge, E. A. CCDC90A (MCUR1) is a cytochrome coxidase assembly factor and not a regulator of the mitochondrial calcium uniporter. Cell Metab. 21, 109-116 (2015).

43. Smith, R. A. J., Porteous, C. M., Gane, A. M. \& Murphy, M. P. Delivery of bioactive molecules to mitochondria in vivo. PNAS 100, 5407-5412 (2003).

44. Powell, R. D., Goodenow, D. A., Christmas, B. A., McKillop, I. H. \& Evans, S. L. Effect of systemic triphenylphosphonium on organ function and oxidative stress. Am. Surg. 84, 36-42 (2018).

45. Nijtmans, L. G. J., Henderson, N. S. \& Holt, I. J. Blue Native electrophoresis to study mitochondrial and other protein complexes. Methods 26, 327-334 (2002).

46. Prudent, J. et al. MAPL SUMOylation of Drp1 Stabilizes an ER / Mitochondrial Platform Required for Cell Death Article MAPL SUMOylation of Drp1 Stabilizes an ER / Mitochondrial Platform Required for Cell Death. Mol. Cell 59, 941-955 (2015).

47. Schindelin, J. et al. Fiji - an Open Source platform for biological image analysis. Nat. Methods 9, 1-15 (2019).

48. Nagashima, S. et al. Golgi-derived PI(4)P-containing vesicles drive late steps of mitochondrial division. Science (80-. ). 1371, 1366-1371 (2020).

49. Mastronarde, D. N. Automated electron microscope tomography using robust prediction of specimen movements. J. Struct. Biol. 152, 36-51 (2005).

50. Saxton, W. O., Baumeister, W. \& Hahn, M. Three-dimensional reconstruction of imperfect two-dimensional crystals. Ultramicroscopy 13, 57-70 (1984).

51. Mastronarde, D. N. Dual-Axis Tomography : An Approach with Alignment Methods That Preserve Resolution. J. Struct. Biol. 120, 343-352 (1997).

\section{ACKNOWLEDGEMENT:}

We would like to thank Dr. Erika Fernandez-Vizarra and Dr. Hiran Prag, both of whom provided feedback on an earlier version of the manuscript. Any errors in this work are the author's own and should not reflect in any way on the contributions from these valued supporters.

\section{AUTHOR CONTRIBUTIONS:}

JT and AK conceived the study, analyzed and visualised data; AK, LT, FT, VŠ, TB performed experiments and analyzed data; JT, JP, MV, JN contributed to methods; all co-authors participated in writing and reviewing the manuscript.

\section{FUNDING:}

This work was supported by PROGRES-Q36-METAB-GAP UK from Charles University, and by the Medical Research Council, UK (MC_ UU_00015/7) for JP. The electron microscopy (Biology Centre, 
bioRxiv preprint doi: https://doi.org/10.1101/2021.03.09.434520; this version posted May 18, 2021. The copyright holder for this preprint (which was not certified by peer review) is the author/funder, who has granted bioRxiv a license to display the preprint in perpetuity. It is made available under aCC-BY-NC-ND 4.0 International license.

České Budějovice) analyses were supported by the MEYS CR (LM2018129 Czech-Biolmaging) and ERD Fund (003/0000441 and CZ.02.1.01/0.0/0.0/16_013/0001775). LT is an MRC-funded PhD student.

\section{COMPETING INTERESTS:}

The authors declare no competing interests. 